\title{
Evaluation of antiarthritic activity of ginkgolic acid against Freund's adjuvant induced arthritic rat model
}

\author{
Ziqiang Zhang,"\#, Xiaogang Wang ${ }^{1, \#, ~ P e n g s h a n ~}$ Li $^{1}$, Minghua Bai ${ }^{1}$, Wenbing Qi ${ }^{\oplus 1^{*}}$ \\ ${ }^{1}$ Department of Orthopedic, Shaanxi Baoji Traditional Chinese \\ Medical Hospital, Shaanxi, People's Republic of China
}

\begin{abstract}
This study aimed to analyze the antiarthritic activity of ginkgolic acid against the Complete Freund's Adjuvant (CFA)-induced arthritis in rats. Arthritis was induced through an intradermal injection of CFA $(0.1 \mathrm{~mL})$ at the right hind footpad of adult Wistar Albino rats. Ginkgolic acid was administered orally at doses of $25 \mathrm{mg} / \mathrm{kg}$ and $50 \mathrm{mg} / \mathrm{kg}$, respectively, once daily via gavage for 25 days upon inducing arthritis. Indomethacin was administered orally at a dose of $3 \mathrm{mg} / \mathrm{kg}$ twice in a week which served as positive control group. The animals were sacrificed and subjected to biochemical and histopathological analysis upon completion of treatment. Ginkgolic acid was able to reverse the arthritic effect $(\mathrm{p}<0.01)$ induced by CFA in a dose dependent manner. Swelling of paw, thymus and spleen index, serum biomarker levels, and pro-inflammatory cytokines were significantly reduced $(\mathrm{p}<0.01)$ by the acid whereas the antioxidant enzyme activities were remarkably restored. The histopathological findings were in agreement with the biochemical results. The results indicate that the antioxidant and anti-inflammatory properties of ginkgolic acid can be credited to the antiarthritic effects, and it can be promoted as a potential agent for therapeutic use against osteoarthritis.
\end{abstract}

Keywords: Ginkgolic acid. Complete Freund's Adjuvant (CFA). Osteoarthritis. Antioxidant. Antiinflammatory. Histopathology.

\section{INTRODUCTION}

Osteoarthritis is a degenerative ailment which is usually linked to aging but can also be linked to other factors like mechanical stress, genetic inheritance, hormonal imbalance, and injury at the bone cartilage, leading to disability and severe pain at the joints (Pan et al., 2017). Characteristics of osteoarthritis include loss of the articular cartilage and damage to the tissues around the joints, degeneration and changes to the subchondral bone which causes chronic pain and limits the function of the joint concerned (Jeong et al., 2015). Inflammatory response has been linked to the progression of osteoarthritis, as recent reports reveal that chondrocytes

*Correspondence: W. Qi, Department of Orthopedic, Shaanxi Baoji Traditional Chinese Medical Hospital, No. 43, Baofu Road, Baoji, Shaanxi, 721000, People's Republic of China, Tel: +8615091080233 . E-mail: sxbj_ qwb@sina.com

\# Co-first Authors : Ziqiang Zhang and Xiaogang Wang contributed equally to this work could respond to inflammatory cytokines to trigger the disease progression (Rajaram, Reddy, Sekhar, 2015). Pro-inflammatory cytokines such as TNF- $\alpha$, IL-1 $\beta$, and IL-6 are known to play pivotal roles in the manifestation of osteoarthritis with high expressions at synovial fluids, causing the pathological condition (Jin et al., 2018). Injury to the tissues surrounding the cartilage worsens the osteoarthritis condition since the inflammatory mediators cause lipid peroxidation of cellular membrane due to oxidative stress. The prevention of inflammation and oxidative stress could be targeted for therapeutic approaches against osteoarthritis.

Non-steroidal anti-inflammatory drugs are among the clinical treatments for osteoarthritic conditions but these drugs have shown adverse side effects such as gastrointestinal problems, renal complaints, cardiovascular troubles, and comorbidities in patients. Therefore a safer alternative therapy is always preferred to handle the problem of osteoarthritis (Lin et al., 2014). Antioxidants from natural products which are well- 
known for various pharmacological uses also possess strong anti-inflammatory properties. Ginkgolic acid is a natural phenolic antioxidant found in the leaves and fruits of Ginkgo biloba L. (Ginkgoaceae). G. biloba L. is a bestselling medicinal herb with known pharmacological properties for cardiovascular ailments, cancer prevention, Alzheimer's disease, as anti-depressants, and many more diseases (Ndjoko, Wolfender, Hostettmann, 2000). Ginkgolic acid is an important active constituent of the G. biloba tree besides other major phytocompounds like flavonoids and terpenes. This acid has been reported to possess anti-microbial activities, anti-tumor properties, and recently its direct inhibition of E1 enzyme (SAE1/ SAE2) sumoylation has proven its ability in suppressing cancer cell migration and growth (Liu et al., 2018). The anti-inflammatory properties of ginkgolic acid have not been evaluated on arthritic animal models. Therefore this study aims to analyze the antiarthritic activity of ginkgolic acid against the Complete Freund's Adjuvant (CFA)-induced arthritis in rats.

\section{MATERIAL AND METHODS}

\section{Chemicals}

Ginkgolic acid and CFA were purchased from Sigma Aldrich, US. Chemicals, reagents, and ELISA commercial kits used for biochemical analysis and enzymatic assays were obtained from BioSource International Inc., US. All other chemicals and reagents were of analytical grade and purchased from authentic suppliers.

\section{Animals}

Forty adult male Wistar Albino rats $(190-210 \mathrm{~g})$ were obtained from the laboratory animal center of our institute. The animals were acclimatized to a 12 hour light/dark cycle at room temperature with free access to water and food prior to the experiment. The Animal Ethical Approval for this experiment was obtained from the Shaanxi Baoji Traditional Chinese Medical Hospital's Ethical Committee (JXC20180105). All animals were treated in humane manner following the guidelines of National Institute of Health and the ethical committee.

\section{Experimental design}

The rats were divided into five groups $(\mathrm{n}=8)$; Group I (normal control), Group II (arthritis control),
Group III \& IV (experimental osteoarthritis groups), and Group V (positive drug control). On day one, arthritis was induced through an intradermal injection of Complete Freund's Adjuvant (CFA) $(0.1 \mathrm{~mL})$ at the right hind footpad of all animal groups except Group I. The animals of Group III \& IV were orally administered ginkgolic acid at doses of $25 \mathrm{mg} / \mathrm{kg}$ and $50 \mathrm{mg} / \mathrm{kg}$ b.w., respectively for 25 consecutive days. Group V animals were orally administered standard drug indomethacin ( $3 \mathrm{mg} / \mathrm{kg}$ b.w.) twice a week till the end of the experiment. The right hind paw volume was measured using a Plethysmometer 37140 (UgoBasile, Italy) and the arthritic index score was measured from 0 - 25 days at every 5 days interval. After 25 days of treatment, the animals were anaesthetized to collect blood samples for serum hematological and biochemical analysis. Animals were sacrificed and right hind ankle joint sections were removed and fixed in $10 \%$ formalin for histopathological analysis. The thymus and spleen index were also estimated and expressed as the ratio of wet organ weight to body weight.

\section{Arthritic index scoring}

The arthritic index scoring was done by determining the characteristics of swelling, redness, erythema, and the paws were checked for severity of the condition. Scores were assigned according to the following scale; 0 = no erythema or swelling, $1=$ swelling or erythema or toes joints, 2 swelling or erythema of toes joints and toes, $3=$ swelling from ankle to toe joints, $4=$ severe swelling from ankle joint up to entire paw (Pan et al., 2017). The arthritic index scores were given as a total of four limbs and the maximum score was 16 .

\section{Biochemical analysis}

The levels of serum alanine transaminase (SGPT), aspartate transaminase (SGOT), and alkaline phosphatase (ALP) were analyzed using commercial kit autoanalyzer (Shimadzu CL-7200, Japan). The levels of inflammatory cytokines TNF- $\alpha$, IL-6, IL-1 $\beta$, and IL-10 were analyzed using commercially available enzyme linked immunosorbent assay (ELISA) kits (Shimadzu, Japan) following the manufacturer's instructions. Concentrations of antioxidant enzymes catalase (CAT), superoxide dismutase (SOD), and serum malondialdehyde (MDA) and reduced glutathione (GSH) levels were assayed using commercial kits (Sigma Diagnostic kit, 
St. Louis, USA). The total protein concentration was determined following Bradford method, using crystalline serum bovine albumin as standard.

\section{Histopathological analysis}

Ankle joint sections fixed in $10 \%$ formalin were dehydrated with gradient of ethanol fixed in paraffin, sectioned to $5 \mu \mathrm{m}$ and stained with hematoxylin and eosin (H\&E). The sections were analyzed and imaged under light microscope $(100 \times$ magnification).

\section{Statistical analysis}

Data are expressed as mean \pm S.E.M. Statistical analysis was carried out using one-way ANOVA followed by Tukey's multiple comparison test. $\mathrm{P}$ values less than 0.05 and $0.01(\mathrm{p}<0.05 ; \mathrm{p}<0.01)$ were considered significant. Statistical analysis was performed using SPPS software 17.0 (Chicago, USA).

\section{RESULTS}

\section{Effect of ginkgolic acid on the body weight, hind paw volume, and arthritic score in rats}

According to the results, CFA caused a heavy loss $(p<0.05)$ of body weight in experimental rats compared to the normal rats as shown in Figure 1A. Similarly, the spleen and thymus index were also increased $(p<0.05)$ in arthritis induced Group II rats compared to the normal control Group I as shown in Figure 1B. However, these results were reversed by the administration of ginkgolic acid which significantly preserved $(p<0.01)$ the body weight of rats in Group III and IV and reduced the organ index compared to CFA-induced rats.

Hind paw volume was seen to be elevated $(\mathrm{p}<0.05)$ over time in the arthritic rats of Group II compared to normal rats over a 25 days period. In contrast, treatment or rats of Group III and IV with ginkgolic acid prevented the swelling of hind paw volume $(\mathrm{p}<0.01)$ over time as shown in Figure 1C. The arthritic index score was significantly high $(p<0.05)$ in CFA-treated rats of Group II compared to normal control rats in Group I as shown in Figure 1D. Ginkgolic acid administration at doses of $25 \mathrm{mg} / \mathrm{kg}$ and $50 \mathrm{mg} / \mathrm{kg}$ b.w., respectively significantly reduced $(\mathrm{p}<0.01)$ the arthritic index score in 25 days, compared to the arthritic rats of Group II. The positive control rats in Group V exhibited slightly better recovery than the rats in Group IV administered high dose treatment of ginkgolic acid

\section{Effects of ginkgolic acid on the levels of RBCs, WBCs, $\mathrm{Hb}$ and serum enzymes in arthritic rats}

Levels of red blood cells (RBC), white blood cells (WBC), and hemoglobin ( $\mathrm{Hb}$ ) in CFA-induced arthritic rats were estimated. The values of $\mathrm{RBC}$ and $\mathrm{Hb}$ were significantly lowered $(p<0.05)$ in CFA-induced arthritic rats of Group II compared to normal rats in Group I. The value of WBC was highly elevated $(p<0.05)$ in arthritic rats of Group II compared to the control rats. These results were ameliorated by ginkgolic acid ( $\mathrm{p}<$ 0.01 ) towards normal in a dose dependent manner as shown in Table I. High dose treatment of ginkgolic acid showed almost similar results to the positive control rats in Group V.

The levels of serum enzymes SGOT, SGPT, and ALP were significantly increased $(p<0.05)$ in the CFA-induced arthritic rats of Group II compared to the control rats. The administration of ginkgolic acid dose dependently reduced $(p<0.01)$ the levels of the serum enzymes as shown in Figure 2.

TABLE I - Effect of ginkgolic acid on RBCs, WBCs and $\mathrm{Hb}$ in CFA-induced arthritis in rats

\begin{tabular}{lccc}
\hline GROUPS & $\begin{array}{c}\mathbf{R B C} \\
\left(\times \mathbf{1 0}^{6} / \boldsymbol{\mu L}\right)\end{array}$ & $\begin{array}{c}\mathbf{W B C} \\
\left(\times \mathbf{1 0}^{3} / \boldsymbol{\mu L} \mathbf{L}\right)\end{array}$ & $\begin{array}{c}\mathbf{H b} \\
(\mathbf{g} / \mathbf{d l})\end{array}$ \\
\hline Group I & $9.46 \pm 0.75$ & $11.24 \pm 0.39$ & $13.72 \pm 0.77$ \\
Group II & $6.31 \pm 0.92^{\#}$ & $22.45 \pm 0.48^{\#}$ & $5.65 \pm 0.48^{\#}$ \\
Group III & $7.24 \pm 0.63^{* *}$ & $17.87 \pm 0.63^{* *}$ & $9.36 \pm 0.52^{* *}$ \\
Group IV & $7.98 \pm 0.37^{* *}$ & $12.23 \pm 0.29^{* *}$ & $11.58 \pm 0.65^{* *}$ \\
Group V & $8.15 \pm 0.46^{* *}$ & $12.05 \pm 0.83^{* *}$ & $12.28 \pm 0.91^{* *}$ \\
\hline
\end{tabular}

Values expressed as mean \pm SEM $(n=8)$ and analyzed by one-way ANOVA followed by Tukey's multiple comparisons test. $\# \mathrm{p}<0.05$ as compared to normal control group I. $* * \mathrm{p}<$ 0.01 as compared to CFA group II. 

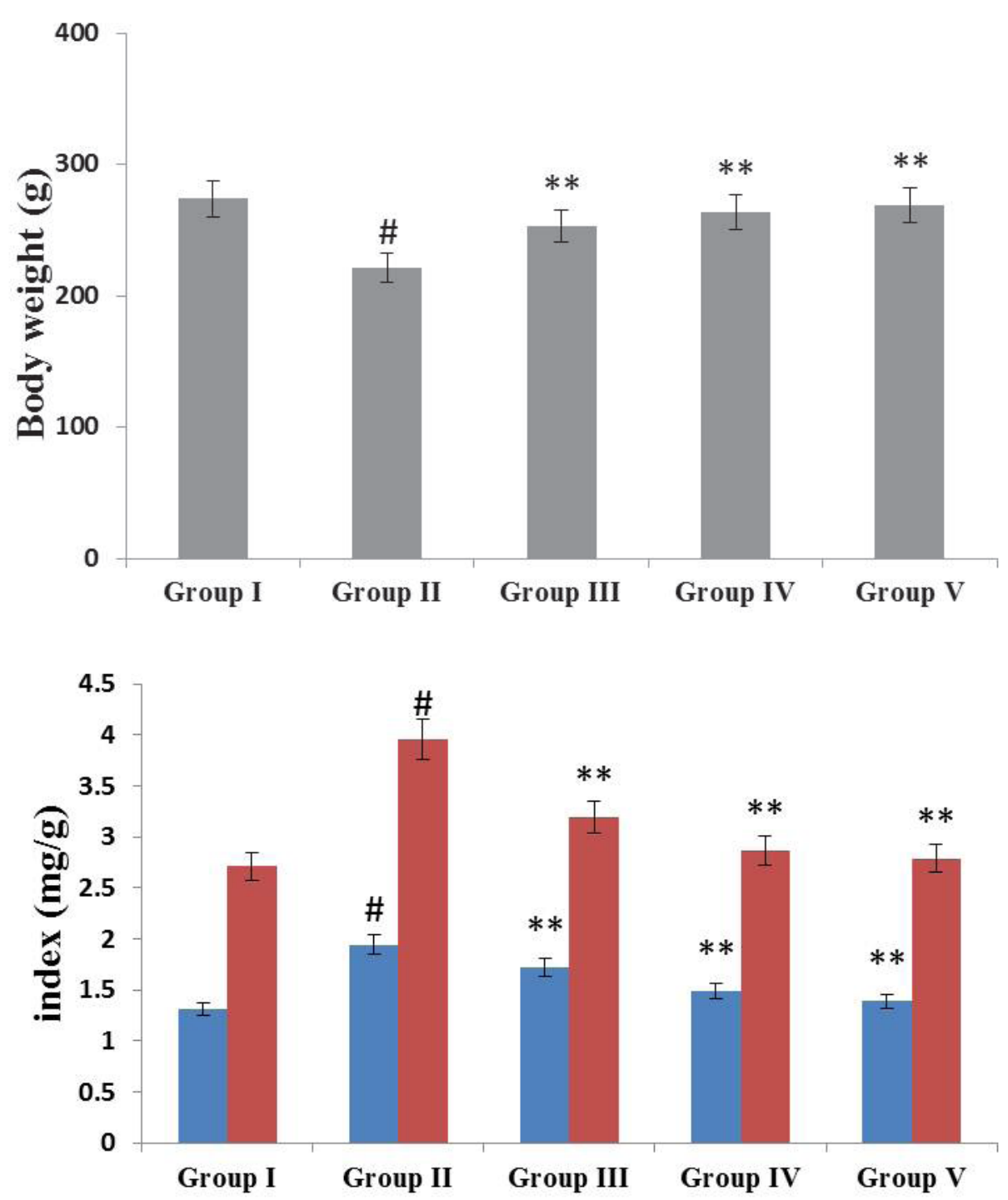

- thymus index $\quad$ spleen index

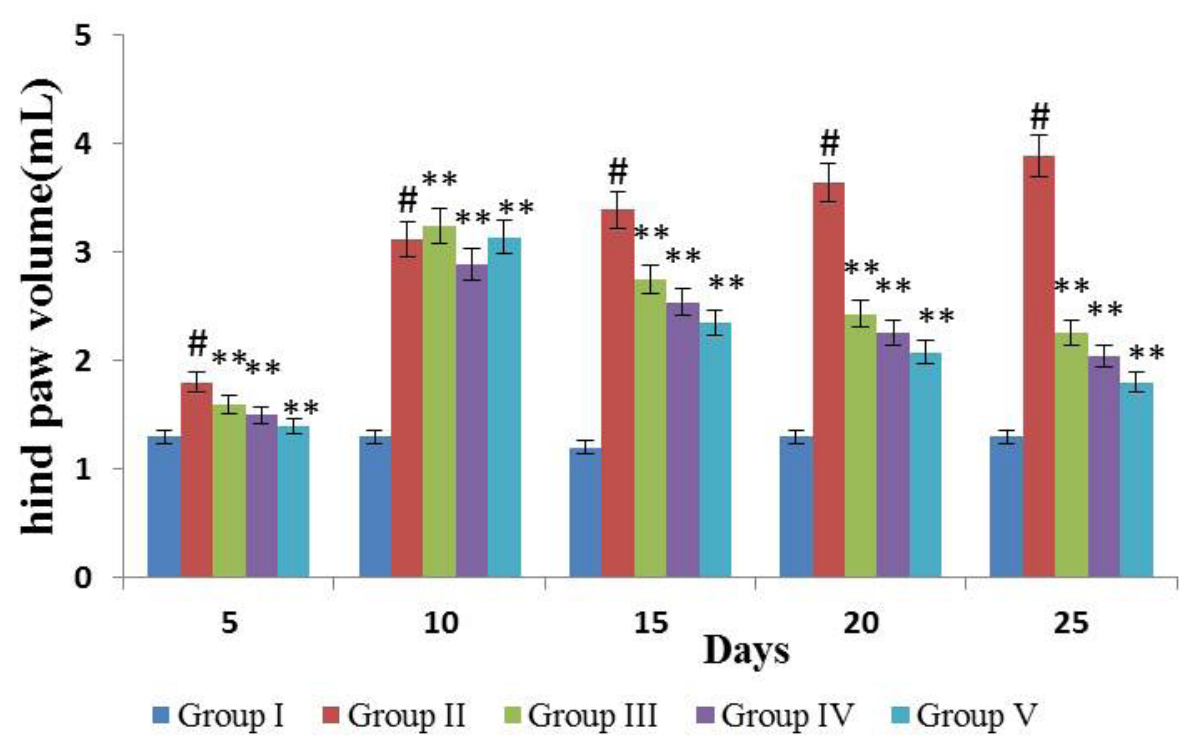




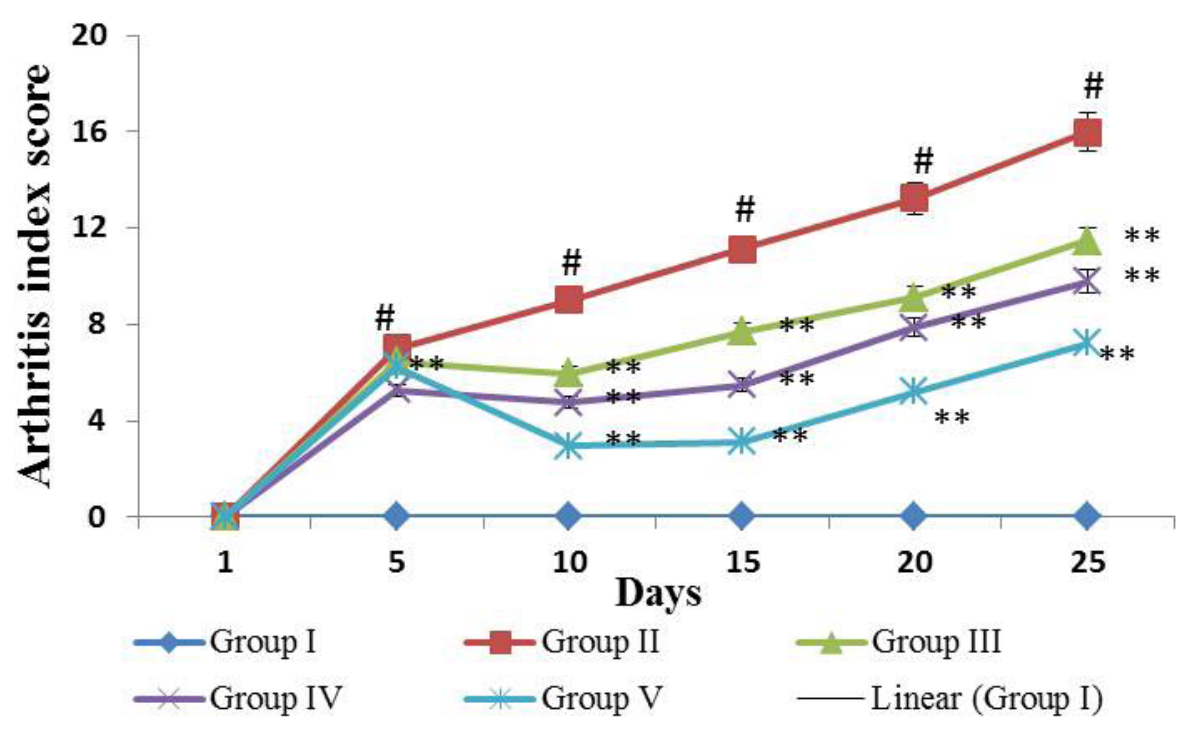

FIGURE 1 - Effect of ginkgolic acid on CFA induced osteoarthritis rats. (A) Effect on body weight; (B) Effect on organ indexes; (C) Effect on hind paw volume; (D) Effect on arthritis index score. Values expressed as mean \pm SEM $(n=8)$ and analyzed by one-way ANOVA followed by Tukey's multiple comparisons test. \# $\mathrm{p}<0.05$ as compared to normal control group I. ** $\mathrm{p}<0.01$ as compared to CFA group II.

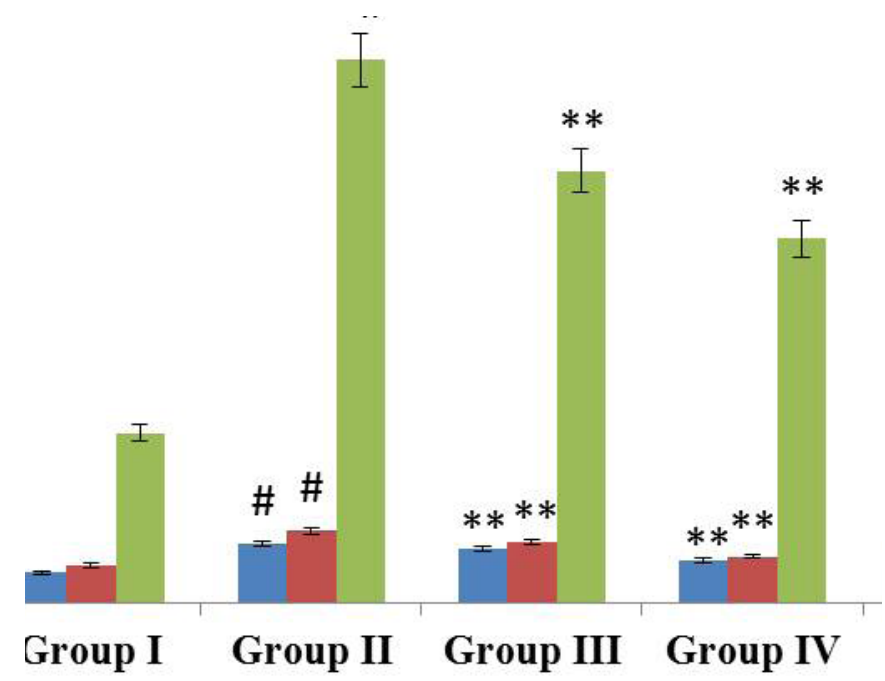

FIGURE 2 - Effect of ginkgolic acid on SGOT, SGPT and ALP enzymes in CFA-induced arthritic rats. Values expressed as mean \pm SEM $(n=8)$ and analyzed by one-way ANOVA followed by Tukey's multiple comparisons test. \# $\mathrm{p}<0.05$ as compared to normal control group I. $* * \mathrm{p}<0.01$ as compared to CFA group II.

\section{Effects of ginkgolic acid on the levels of inflammatory cytokines in CFA-induced arthritic rats}

The levels of pro-inflammatory cytokines TNF- $\alpha$, IL-6, and IL-1 $\beta$ were significantly elevated $(\mathrm{p}<0.05)$ in the arthritic rats of Group II compared to the normal control rats in Group I. The level of anti-inflammatory cytokine IL-10 was reduced $(\mathrm{p}<0.05)$ in Group II arthritic rats compared to the normal rats. Ginkgolic acid remarkably reversed $(p<0.01)$ the levels of inflammatory cytokines towards normal levels in animals of Group III and IV as shown in Figure 3A, 3B, 3C, and 3D. 

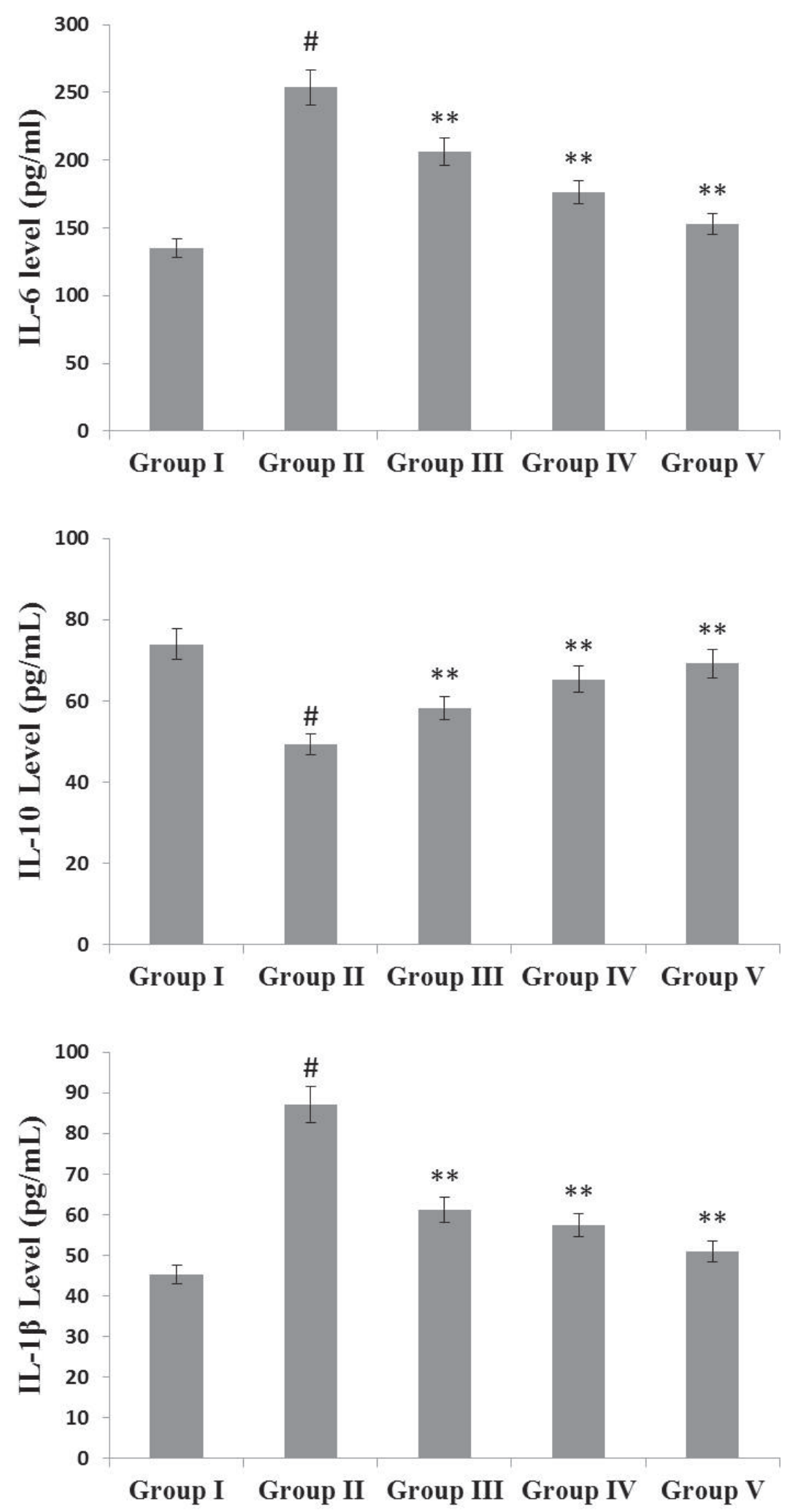

FIGURE 3 - Effect of ginkgolic acid on the levels of inflammatory cytokines in CFA-induced arthritic rats.

(A) Effect on the levels of TNF- $\alpha$; (B) Effect on the levels of IL-6; (C) Effect on the levels of IL-10; (D) Effect on the levels of IL-1 $\beta$. Values expressed as mean \pm SEM $(n=8)$ and analyzed by one-way ANOVA followed by Tukey's multiple comparisons test. \# $\mathrm{p}<0.05$ as compared to normal control group I. ${ }^{* *} \mathrm{p}<0.01$ as compared to CFA group II. 


\section{Effects of ginkgolic acid on MDA, CAT, SOD,} and GSH levels in CFA-induced arthritic rats

The biochemical parameter for the determination of cellular membrane lipid peroxidation is given as MDA formations. MDA levels were elevated significantly $(\mathrm{p}<$ 0.05 ) in the arthritic rats of Group II as shown in Figure 4A. Administration of ginkgolic acid in rats of Group III and IV caused a reduction in the MDA formations ( $\mathrm{p}<$ 0.01) compared to the arthritic rats in Group II.
The levels of antioxidant enzymes CAT, SOD and non-enzymatic antioxidant GSH were highly reduced $(p<0.05)$ in arthritic rats of Group II compared to normal rats of Group I. In contrast, the administration of ginkgolic acid remarkable restored $(\mathrm{p}<0.01)$ the levels of CAT, SOD, and GSH towards the normal state as shown in Figure 4B. The positive control rats of Group V also showed increased levels of CAT, SOD, and GSH $(\mathrm{p}<0.01)$ compared to the arthritic rats of Group II.
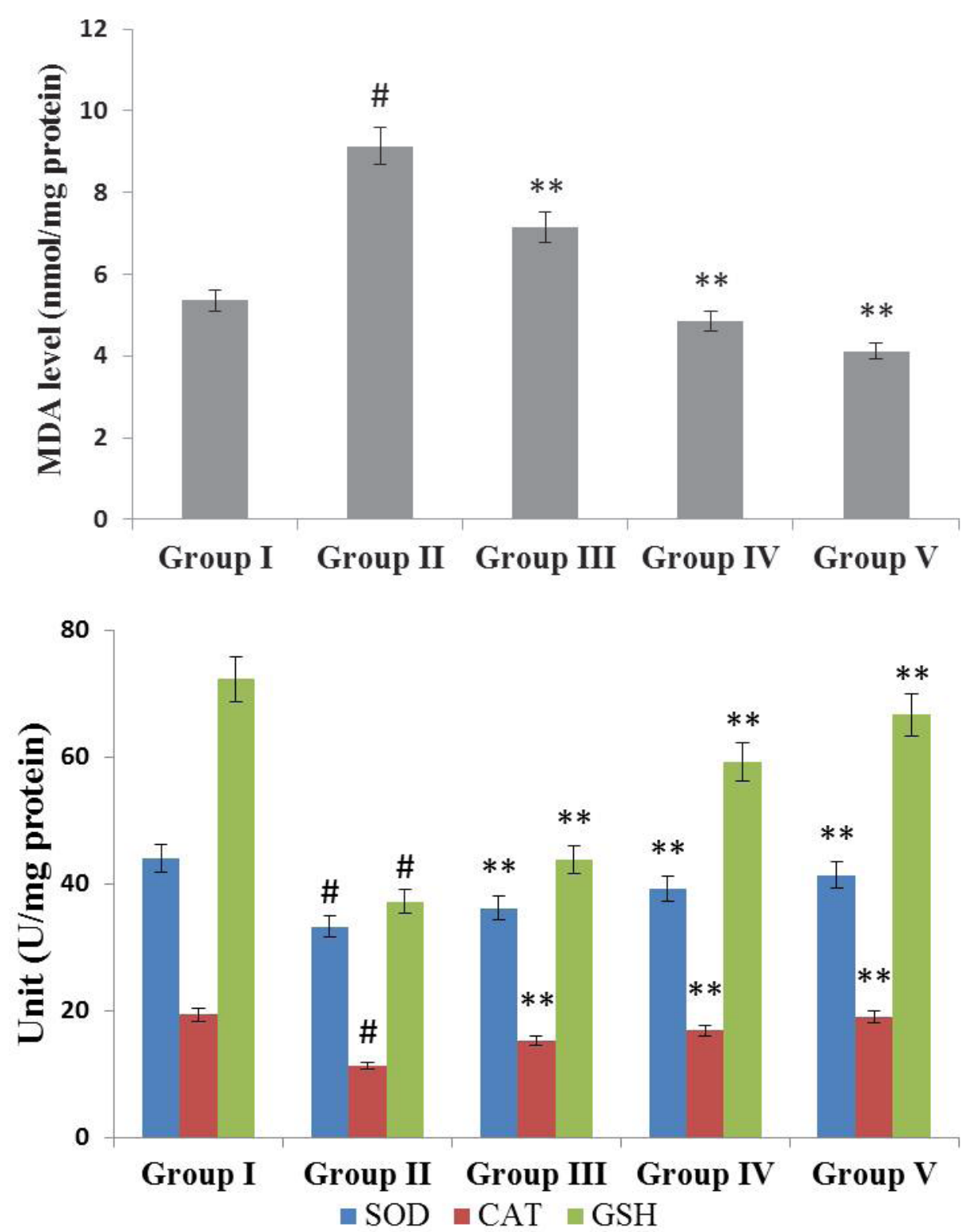

FIGURE 4 - Effect of ginkgolic acid on oxidative stress markers in CFA-induced arthritic rats. (A) Effect on the levels of MDA formation; (B) Effect on the levels of SOD, CAT and GSH. Values expressed as mean \pm SEM $(\mathrm{n}=8)$ and analyzed by oneway ANOVA followed by Tukey's multiple comparisons test. $\# \mathrm{p}<0.05$ as compared to normal control group I. $* *$ p $<0.01$ as compared to CFA group II. 


\section{Effect of ginkgolic acid on the histopathological changes in arthritic rats}

Figure 5 presents the histopathological analyses on the right hind ankle joint of rats. Rats of Group I presented normal histological arrangements. In disparity, the CFA-induced arthritic rats of Group II presented inflammation and osteoarthritic features such as synovial hyperplasia, excessive inflammatory cell infiltrations, abnormal bone cartilage degeneration, and loss of bone marrow. The treatment with ginkgolic acid remarkably reversed the results as shown in rats of Group III, which presented signs of inflammation but the bone cartilage degeneration and synovial lining obliteration was moderate. High dose of ginkgolic acid in Group IV preserved the pathology of hind ankle joint with reduced inflammation and controlled arthritic condition compared to Group II arthritic rats.

\section{Group I}

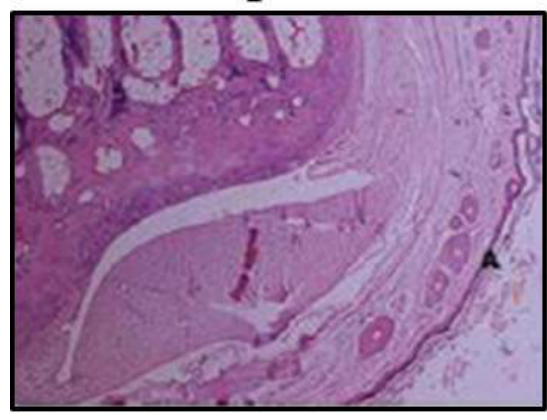

Group IV

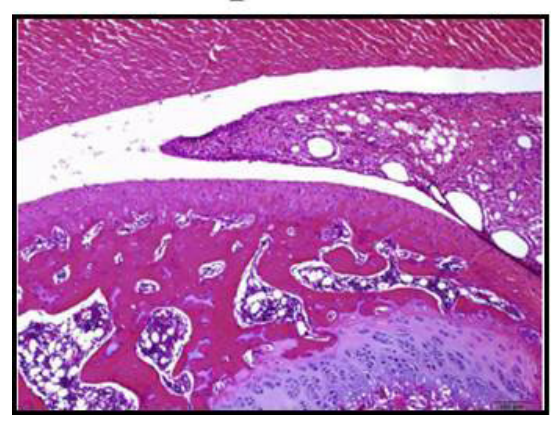

Group II

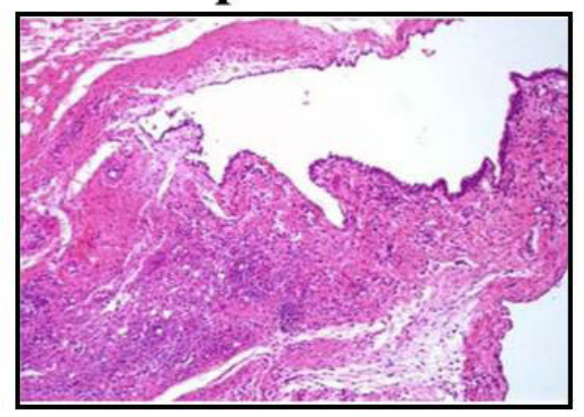

Group V

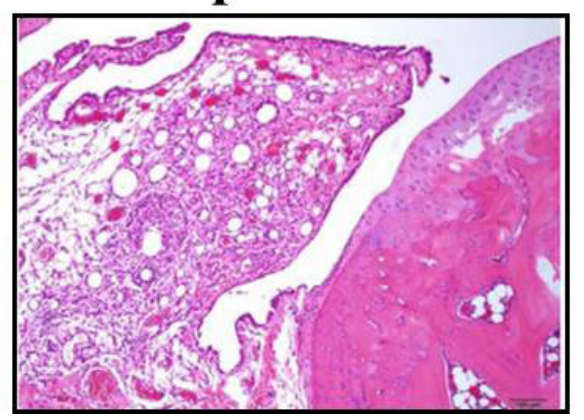

FIGURE 5 - Histopathological alterations by ginkgolic acid on CFA-induced osteoarthritis.

Sections of ankle joints from arthritis control Group II shows severe signs of synovial hyperplasia, excessive inflammatory cell infiltrations, abnormal bone cartilage degeneration, and loss of bone marrow. Ginkgolic acid significantly reversed the arthritic condition as demonstrated by Group III and IV. H\&E staining at 100× magnification.

\section{DISCUSSION}

Osteoarthritis induced with CFA in animal experimental model is often used for pre-clinical pharmacological studies (Rahmati, Mobasheri, Mozafari, 2016). It is characterized by excessive inflammatory reaction, damage or loss of bone cartilage, bone joint injury, synovial hyperplasia, and limb swelling. These features were exhibited by the arthritic control rats in Group II as shown in our histopathological results. Therefore osteoarthritis was clearly induced in our experimental rats. Ginkgolic acid was administered at the doses of $25 \mathrm{mg} / \mathrm{kg}$ and $50 \mathrm{mg} / \mathrm{kg}$ b.w., respectively to rats induced with CFA. From the study, ginkgolic acid 
was able to reverse the effects of CFA induced arthritis in a dose-dependent manner. This was evidenced by the increase in body weight of rats, reduction of organ index and arthritic index score, and reduction in the hind paw volume. Spleen and thymus index observation was noted to evaluate the immunoregulatory effect of ginkgolic acid against CFA-induced arthritis and it was found that the functions of thymus and spleen were protected by ginkgolic acid with an index almost similar to normal rats. The hematological parameters also supported the organ index results where red blood cells and hemoglobin levels were reduced in arthritic rats which relates to the increased weight of thymus and spleen, which acts as filters of dead blood cells. Serum enzymes SGOT, SGPT, and ALP are liver and kidney damage markers which are usually tested for experimental arthritis and drug administration (Zheng et al., 2014). The results indicate that ginkgolic acid was able to reduce the damage caused by CFA as the levels of serum enzymes were significantly lower.

Lipid peroxidation occurs during oxidative stress where excessive free radical production damages the cell membrane, causing MDA formations (Poulet, Staines, 2016). To overcome the problem of free radicals, naturally occurring enzymatic and non-enzymatic antioxidants such as SOD, CAT, and GSH protects the cellular environment by scavenging the reactive free radicals (Zhu et al., 2017). Antioxidants are known to possess free radical scavenging activities which could help in preventing pathological conditions such as osteoarthritis (Wang et al., 2016). This is the reason many naturally derived antioxidants are being studied for their pharmacological properties against various ailments in pre-clinical trials (Chang et al., 2015). From the results, it was shown that there was an increase in MDA levels in CFA-induced arthritis in rats, clearly indicating that oxidative stress was triggered. The situation is supported by the loss of the activities of antioxidant enzymes SOD and CAT, whereas the level of GSH was also reduced in arthritic rats. Being a strong antioxidant, ginkgolic acid remarkably mitigated the oxidative stress condition by replenishing the levels of GSH, SOD, and CAT and also prevented lipid peroxidation as exhibited by reduced MDA formations.

Inflammation is commonly associated with the development of osteoarthritis due to the presence of inflammatory cytokines and other relevant mediators (Wang et al., 2017). The release of inflammatory cytokines is controlled by the regulation of NF- $\kappa \mathrm{B}$ signaling pathway which is commonly associated with lipid peroxidation (Hou, Hou, Liu 2017). Proinflammatory cytokines such as TNF- $\alpha$, IL-6, and IL-1 $\beta$ are the main precursors of inflammation (Lima-Garcia et al., 2011). The results clearly show that CFA-induced arthritis in rats increased the levels of pro-inflammatory cytokines TNF- $\alpha$, IL-6, and IL-1 $\beta$ whereas the level of anti-inflammatory cytokine IL-10 was reduced. The inflammatory process was observed in the arthritic rats from the swelling of hind paw, increase in white blood cells, excessive infiltration of lymphocytes as exhibited by the histopathological findings, and the arthritic index. Inflammation of the ankle joints due to CFA-induced inflammation must have contributed to the development of osteoarthritis in rats. However, ginkgolic acid was able to reduce the levels of pro-inflammatory cytokines and elevate the anti-inflammatory cytokine which contributes to the anti-inflammatory effect of ginkgolic acid. It can be said that ginkgolic acid is able to suppress the activation of NF- $\kappa \mathrm{B}$ signaling pathway through prevention of lipid peroxidation.

\section{CONCLUSION}

Collectively, CFA-induced osteoarthritis in rats was notably altered by the oral administration of ginkgolic acid in a dose dependent manner. The biochemical results are in agreement with the histopathological findings. Anti-arthritic properties of ginkgolic acid can be attributed to its ability to prevent lipid peroxidation, elevate the enzymatic and non-enzymatic antioxidants, prevent the infiltration of inflammatory cytokines, and increase the level of anti-inflammatory cytokine IL-10. Prevention of osteoarthritis can be achieved through inhibition of inflammation at the site. Therefore, ginkgolic acid can be recommended as a potential compound with anti-inflammatory, anti-arthritic, and therapeutic effects for osteoarthritis. Future studies should be conducted on the detailed mechanisms involved in its anti-inflammatory effects.

\section{CONFLICT OF INTEREST}

There is no conflict of interest.

\section{REFERENCES}

Chang X, He H, Zhu L, Gao J, Wei T, Ma Z, Yan T. Protective effect of apigenin on Freund's complete adjuvant-induced 
arthritis in rats via inhibiting $\mathrm{P} 2 \mathrm{X} 7 / \mathrm{NF}-\mathrm{\kappa B}$ pathway. ChemBiol Inter. 2015;236:41-6.

Hou S, Hou C, Liu J. CX3CL1 promotes MMP-3 production via the CX3CR1, c-Raf, MEK, ERK, and NF-кB signaling pathway in osteoarthritis synovial fibroblasts. Arth Res Ther. 2017;19(1):282.

Jeong YJ, Kim I, Cho JH, Park DW, Kwon JE, Jung MW, et al. Anti-osteoarthritic effects of the Litsea japonica fruit in a rat model of osteoarthritis induced by monosodium iodoacetate. Plos One. 2015;10(8):e0134856.

Jin R, Shen M, Wu R, Xu S. Acamprosate protects against adjuvant-induced arthritis in rats via blocking the ERK/ MAPK and NF- $\mathrm{BB}$ signaling pathway. Inflammation. 2018;41(4):1194-1199.

Lima-Garcia JF, Dutra RC, da Silva K, Motta EM, Campos MM, Calixto JB. The precursor of resolvin D series and aspirin-triggered resolvin D1 display anti-hyperalgesic properties in adjuvant-induced arthritis in rats. Brit $\mathrm{J}$ Pharmacol. 2011;164(2):278-93.

Lin B, Zhao Y, Han P, Yue W, Ma X, Rahman K, et al. Anti-arthritic activity of Xanthium strumarium L. extract on complete Freund's adjuvant induced arthritis in rats. J Ethnopharmacol. 2014;155(1):248-55.

Liu H, Li J, Lu D, Li J, Liu M, He Y, et al. Ginkgolic acid, a sumoylation inhibitor, promotes adipocyte commitment but suppresses adipocyte terminal differentiation of mouse bone marrow stromal cells. Sci Rep. 2018;8(1):2545.

Ndjoko K, Wolfender J, Hostettmann K. Determination of trace amounts of ginkgolic acids in Ginkgo biloba L. leaf extracts and phytopharmaceuticals by liquid chromatography-electrospray mass spectrometry. J Chrom B: Biomed Sci Appl. 2000;744(2):249-55.
Pan T, Cheng TF, Jia YR, Li P, Li F. Anti-rheumatoid arthritis effects of traditional Chinese herb couple in adjuvant-induced arthritis in rats. J Ethnopharmacol. 2017;205:1-7.

Poulet B, Staines KA. New developments in osteoarthritis and cartilage biology. Curr Op Pharmacol. 2016;28:8-13.

Rahmati M, Mobasheri A, Mozafari M. Inflammatory mediators in osteoarthritis: A critical review of the stateof-the-art, current prospects, and future challenges. Bone. 2016;85:81-90.

Rajaram C, Reddy KR, Sekhar KB. Evaluation of antiarthritic activity of Caesalpinia pulcherrima in Freund's complete adjuvant induced arthritic rat model. J Young Pharm. 2016;7(2):128-32.

Wang S, Abramson S, Attur M, Karsdal M, Preston R, Lozada $\mathrm{C}$, et al. Safety, tolerability, and pharmacodynamics of an anti-interleukin- $1 \alpha / \beta$ dual variable domain immunoglobulin in patients with osteoarthritis of the knee: A randomized phase 1 study. Osteoarthr Cart. 2017;25(12):1952-61.

Wang S, Wang Y, Liu X, Guan L, Yu L, Zhang X. Antiinflammatory and anti-arthritic effects of taraxasterol on adjuvant-induced arthritis in rats. J Ethnopharmacol. 2016;187:42-8.

Zheng $\mathrm{C}$, Zhao $\mathrm{X}$, Ai $\mathrm{H}$, Lin $\mathrm{B}$, Han $\mathrm{T}$, Jiang $\mathrm{Y}$, et al. Therapeutic effects of standardized Vitex negundo seeds extract on complete Freunds adjuvant induced arthritis in rats. Phytomed. 2014;21(6):838-46.

Zhu M, Li M, Zhang L, Huang Y, Lu Y, Wang T, Fu F. Ginsenoside Rg1 attenuates adjuvant-induced arthritis in rats via modulation of PPAR- $\gamma / \mathrm{NF}-\kappa \mathrm{B}$ signal pathway. Oncotarget. 2017;8(33):55384-93.

Received for publication on $08^{\text {th }}$ August 2018 Accepted for publication on $02^{\text {nd }}$ December 2018 